\title{
Um Protocolo MAC para Redes de Sensores sem Fio Voltado para Aplicações Específicas Utilizando o Método de Acesso TDMA
}

\author{
Sandro Silva de Oliveira e Shusaburo Motoyama
}

\begin{abstract}
Resumo - Neste artigo é apresentado um novo protocolo MAC para redes de sensores sem fio baseado no método de acesso TDMA (Time Division Multiple Access). É voltado para aplicações que possuem um pequeno número de sensores altamente concentrados em uma pequena região. A principal característica do protocolo proposto é o agendamento de transmissão simplificado que garante um baixo consumo de energia e um overhead reduzido. $O$ consumo de energia e $o$ atraso de espera dos pacotes do protocolo proposto são estimados analiticamente $\mathrm{e}$ comparado com outros protocolos. Os resultados mostram que o protocolo é bastante adequado para a finalidade proposta tanto em consumo de energia como em controle de atraso de pacotes.

Palavras chave - Redes de sensores sem fio, protocolos de controle de acesso ao meio, Acesso múltiplo por divisão de tempo, redução de consumo de energia, atraso.
\end{abstract}

Abstract - In this article a new MAC protocol for wireless sensor networks based on the TDMA (Time Division Multiple Access) access method is proposed. The proposed protocol is intended for applications which the sensors are highly concentrated in a small region. The main feature of the proposed protocol is the simplified transmission scheduling that guarantees a low consumption of energy and a reduced overhead. The energy consumption and packet waiting delay of the proposed protocol are analytically estimated and compared to others protocols. The results have showed that the protocol is well fitted for proposed purpose in energy consumption as well as in packet delay control.

Keywords - Wireless sensor networks, Medium access control (MAC) protocols, Time division multiple access (TDMA), Reduction of energy consumption, Packet delay.

\section{INTRODUÇÃO}

As Redes de Sensores Sem Fio (RSSFs) são constituídas de um grande número de nós distribuídos que colaboram uns com os outros para executarem tarefas. Um nó é representado por um pequeno hardware, composto de um ou mais sensores, um rádio transmissor/receptor, bateria e um processador. Este tipo de rede tem sido objeto de intensas pesquisas aplicadas a diferentes cenários, como monitoramento de ambientes, robótica e sistemas médicos.

Os nós das RSSFs podem estar localizados em ambientes de difíceis acessos, dificultando a manutenção e recarga da bateria. Assim, o eficiente consumo de energia é de extrema importância para o tempo de vida da rede de sensores. Vários

Sandro Silva de Oliveira e Shusaburo Motoyama, Departamento de Telemática da Faculdade de Engenharia Elétrica e Computação - FEEC, da Universidade Estadual de Campinas - UNICAMP. End.: Cidade Universitária Zeferino Vaz, Barão Geraldo, Campinas, São Paulo - Brasil, CEP: $13083-$ 970. E-mail: sandro@dt.fee.unicamp.br, motoyama@dt.fee.unicamp.br. Este trabalho é financiado pela CAPES. critérios são propostos para minimizar o consumo de energia, como [1], [7], [9]: evitar colisão, diminuir o overhearing (escuta inútil), controle do overhead e idle listening (escuta ociosa, quando não há tráfego na rede). Nas arquiteturas de protocolos das RSSFs, uma das camadas de maior relevância é a MAC (Medium Access Control). Os métodos de controle de acesso ao meio influenciam no consumo de energia dos dispositivos de rede, na forma como as informações são encaminhadas e na maneira como as aplicações são concebidas. Vários protocolos MAC para RSSFs têm sido propostos na literatura [1], [2], [6], visando obter eficientes esquemas de acesso, e são baseados em contenção ou então em TDMA.

Os protocolos MAC propostos em [1], [2] são baseados no esquema de acesso com contenção e usam um período cíclico de tempo. Cada período é dividido em uma parte ativa e uma de repouso em que o nó é desativado. Na parte ativa todos os nós são acordados e executam toda a comunicação de troca de informações necessária, iniciando com o diálogo RTS-CTS (Request-To-Send/Clear-To-Send) para controle da contenção e em seguida para transmissão de dados e recepção do ACK (mensagem de confirmação). É o mesmo protocolo de controle usado no MAC do IEEE 802.11. A diferença entre os protocolos propostos em [1], [2] está que no primeiro a parte ativa assim como a de repouso é fixa e no segundo, esta é variável, o que o torna mais eficiente. Um protocolo denominado TRAMA baseado em TDMA para RSSFs é proposto em [5]. Nesse protocolo um quadro é dividido na parte de agendamento e na parte de dados. A parte de dados é subdividida em slots para transmissão de dados. Uma desvantagem deste protocolo é que o algoritmo de agendamento é baseado em um esquema de eleição para escolher quais nós devem transmitir e receber, o qual é complexo, ocasionando um número excessivo de pacotes de overhead.

Outros protocolos baseados em TDMA são propostos na literatura, como os apresentados em [13] e [14]. O protocolo proposto em [13] atribui slots somente quando quer enviar ou receber informação, caso contrário, mantém o transceptor desligado para evitar a escuta desnecessária dos nós vizinhos. Este protocolo faz uso de um pacote wake-up para ativar os nós quando necessário. Em [14] apresenta-se um outro protocolo baseado em TDMA, voltado para aplicações que solicitam informações periodicamente e com garantia de entrega. Este protocolo também apresenta um esquema de controle dos slots flexível, destinado para redes com um grande número de nós sensores. 
Outros esquemas de acesso apresentados na literatura [3], [4] utilizam algumas técnicas para minimizar o consumo de energia. Em [3] o controle de acesso ao canal é feito por um esquema de amostragem de ruído do canal de transmissão, determinando assim o estado do mesmo. Já em [4] é proposto um algoritmo distribuído para o balanceamento da carga da rede, estabelecendo que os nós sensores com estado de energia baixa devem ser tratados diferentemente e usados com menor freqüência no encaminhamento das mensagens.

A maioria dos protocolos supracitados são voltados à aplicações gerais e são baseados em contenção [9]. No entanto, para uma aplicação específica de RSSFs o consumo de energia pode ser minimizado usando as características apresentadas pela aplicação. Neste artigo é apresentado um novo protocolo MAC para RSSFs voltado a aplicações específicas, tais como sistemas médicos ou para uma aplicação em que os nós sensores estão altamente concentrados em uma pequena área física. Nesta área a mobilidade dos nós sensores não é alta e o sinal de rádio de cada nó é alcançado por todos outros nós, reduzindo o overhead de comunicação e o consumo de energia de cada nó. O método de acesso do protocolo proposto é baseado em TDMA, sendo semelhante ao protocolo proposto em [6], diferenciando-se somente na forma de agendamento de acesso. Em [6], cada sensor tem uma posição fixa reservada na parte de agendamento e no esquema do protocolo proposto, há um número limitado de slots de tempo que são disputados pelos nós sensores para transmitirem as solicitações de agendamento.

$\mathrm{O}$ artigo está organizado da seguinte forma. Na seção II, são apresentados os principais protocolos MAC para RSSFs encontrados na literatura. O protocolo proposto é descrito na seção III. Na seção seguinte, a estimativa de consumo de energia do protocolo proposto é analisada e comparada com alguns dos protocolos existentes na literatura. $\mathrm{Na}$ seção V é apresentada a análise de atraso espera dos pacotes do protocolo proposto. Finalmente, as principais conclusões são descritas na seção $\mathrm{V}$.

\section{PRINCIPAIS PROTOCOLOS MAC PARA RSSFS}

Há dois tipos principais de protocolos de acesso MAC para RSSFs, aqueles que são baseados em agendamento (scheduling) ou alocação de recursos, representado pelo método TDMA, e aqueles que são baseados em contenção. Os protocolos baseados em TDMA utilizam, em geral, quadros repetitivos, divididos em vários slots de tempo (ou canais). No caso do TDMA tradicional, em cada quadro, cada nó tem o acesso a um slot de tempo dedicado, em que pode enviar e/ou receber dados, utilizando toda a largura de banda do canal. Para utilização do TDMA em RSSFs, a alocação dos canais deve ser dinâmica, cada canal sendo alocado ao um nó somente quando houver dados para transportar. Dessa forma, um quadro do esquema de acesso TDMA para RSSFs, possui uma parte reservada para agendamento dos nós para definir a seqüência de transmissão. Já nos protocolos de contenção baseados no Carrier Sense Multiple Access with Collision Avoidance - CSMA/CA, os nós disputam o acesso ao canal, existindo a probabilidade de colisões no envio de dados. Os protocolos baseados no CSMA/CA necessitam de quadros de controle para estabelecer um diálogo de comunicação entre as estações. Neste método há colisões e um overhead maior. Nesta seção são apresentados alguns dos principais protocolos MAC para RSSFs presentes na literatura.

O S-MAC, protocolo proposto em [1] e baseado em CSMA/CA, utiliza sincronização para ordenação dos modos de operação do rádio. É destinado a aplicações dirigidas a eventos, insensíveis a latência e com baixa taxa de envio de mensagens. O S-MAC usa o método de comunicação baseado em RTS/CTS/DATA/ACK para detectar a portadora física e resolver o problema de colisão. O esquema do S-MAC é baseado em um ciclo de operação dos nós com tempos fixos de escuta (listen) e repouso (sleep). Neste esquema, cada nó irá repousar por um tempo, e então acordará e ficará escutando para ver se tem algum outro nó querendo transmitir informação para ele. A duração do tempo para escuta ou para repouso é selecionada de acordo com a aplicação. O esquema de sincronização entre os nós é realizado enviando um pacote denominado SYNC. Este inclui o endereço do emissor e o tempo do próximo repouso. A desvantagem do S-MAC está na utilização do ciclo de atividade (duty cycle) fixo, não permitindo o controle dinâmico do tempo dos modos de operação de escuta e repouso.

O protocolo T-MAC apresentado em [2] é semelhante ao S-MAC e visa reduzir o tempo de idle listening, utilizando-se de ciclos de atividade e repouso dinâmicos para diminuir o consumo de energia do nó. O controle do tempo ativo é feito por um temporizador chamado $\mathrm{T}_{\mathrm{A}}$ (time-out), que ao seu término coloca o rádio em repouso. $\mathrm{O}$ nó escuta a rede, transmite e recebe dados durante o seu tempo ativo. A recepção de pacotes RTS ou CTS é suficiente para renovar o tempo $\mathrm{T}_{\mathrm{A}}$. $\mathrm{O}$ intervalo de tempo $\mathrm{T}_{\mathrm{A}}$ deve ser suficiente para receber pelo menos o início de um pacote CTS. O valor do $\mathrm{T}_{\mathrm{A}}$ é estimado pelo tamanho do esquema de contenção do RTS/CTS/DATA/ACK, pelo tamanho de um pacote RTS e o tempo de round-trip (tempo entre o final de um pacote RTS e o início de um pacote CTS). A soma destes três parâmetros deve ser maior que $\mathrm{T}_{\mathrm{A}}$. O T-MAC é mais eficiente em consumo de energia que o S-MAC, mas é limitado em largura de banda, não sendo adequado para grandes transferências de dados. O TMAC é indicado para aplicações de pequeno tráfego. Em [8] propõe-se uma modificação no T-MAC que considera o tráfego da rede para determinar o período de listen/sleep visando uma melhor economia de energia e latência.

O protocolo FS-MAC (Fixed Scheduling Medium Access Control), proposto em [6], é baseado no esquema TDMA e tem o formato de quadro mostrado na Fig. 1. A primeira parte é usada para sincronização do quadro e, também, para reconfiguração dos nós sensores, no caso em que os nós sensores parem de funcionar ou no caso em que há inserção de novos nós sensores. Em um quadro são transmitidos os bits de sincronismo, em um outro quadro, alternadamente, transmitem-se os bits de reconfiguração. A segunda parte do quadro é dividida em $N$ mini-slots, e cada um é alocado a um determinado nó, de modo, 
que cada nó conhece exatamente qual a posição dos outros nós. Isso permite uma redução de overhead de sinalização, pois, os nós sensores necessitam trocar menos informações para transmitirem os dados. Cada mini-slot possui informações de identificação do nó (ID) e da quantidade de canais a serem reservados para transmissão de dados. A alocação de cada nó para o seu tempo de slot correspondente é feita pelo nó central ou de forma distribuída e notificada para todos nós usando o espaço destinado aos bits de reconfiguração.

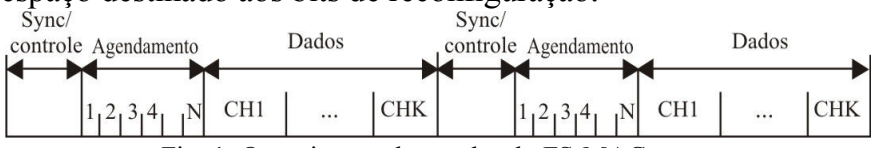

Fig. 1. Organização do quadro do FS-MAC.

A solicitação da reserva do número de canais é feita durante a escrita de identificação. Os mini-slots podem, também, ser utilizados para enviar o sinal de confirmação - ACK. Neste tipo de alocação, cada nó deve estar ativo nesta fase de agendamento. Entretanto, no período do canal de dados, somente os nós transmissores e receptores deverão estar ativos, permitindo que os outros nós fiquem no modo de repouso, economizando energia.

Os projetos de protocolos de acesso de RSSFs para aplicações gerais e para uma rede de grande dimensão requerem análises e implementações de hipóteses bastante genéricas, que talvez nunca sejam utilizadas em uma aplicação específica. Em sua maioria, exceto o protocolo proposto em [6], os protocolos de acesso (MAC) para RSSFs aqui apresentados são desenvolvidos para aplicações gerais e para redes com grande número de nós sensores. Com o objetivo de economizar energia e com um atraso aceitável, explorando características de uma aplicação específica, é proposto neste artigo, um novo protocolo MAC para RSSFs. Na seção a seguir é descrito o protocolo proposto.

\section{DESCRIÇÃO DO PROTOCOLO PROPOSTO SA-MAC}

Em algumas aplicações específicas como em aplicações médicas, a mobilidade dos sensores é limitada a uma pequena área. Desse modo, pode-se utilizar nessas áreas, um nó sensor central e um grupo de sensores de modo que os sensores em cada grupo se comuniquem através desse nó sensor central. A existência de um nó sensor central possibilita uma comunicação mais simples, reduzindo overhead de sinalização (trocas de informação antes da transferência de dados). A comunicação de um sensor de um grupo com sensor de um outro grupo pode ser feita através dos nós sensores centrais. Assim, a rede pode ser expandida com a introdução de vários grupos, tendo cada grupo um nó central. Considerando as características inerentes em aplicações médicas, o esquema de acesso TDMA é mais conveniente, pois, é um esquema que utiliza um nó centralizado e fornece um acesso sem contenção, permitindo desse modo, atrasos reduzidos nas transferências de mensagens.

Na Fig. 2 é mostrada a organização do quadro do protocolo SA-MAC. Como em FS-MAC o quadro é dividido em três partes. A primeira parte é utilizada para o sincronismo de quadro. A segunda parte é utilizada para enviar solicitação de agendamento. Esta parte é dividida em determinado número de slots para que sejam utilizados para solicitar agendamento e, também, para receber confirmações. Como há um número limitado de slots, neste esquema haverá contenções para o acesso a esses slots. Na terceira parte do quadro estão os canais ou slots de tempo para transportar os dados.

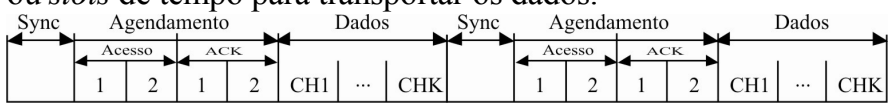

Fig. 2. Formato do quadro do protocolo SA-MAC.

Para ilustrar o funcionamento do SA-MAC, supõe-se a seguinte situação: se um dos nós quiser transmitir, primeiramente, é realizado um sorteio para escolher um dos slots de agendamento para transmitir. Então, a solicitação de agendamento é encaminhada, com informações de identificação do nó transmissor e o número de slots estimado para transmissão, esta informação de agendamento é recebida por todos os nós. Um nó ficará sabendo do sucesso ou falha na transmissão da informação de agendamento somente no quadro seguinte, quando receberá ou não o ACK. Caso ocorra colisão, o nó transmissor disputará novamente o slot de agendamento, até conseguir o acesso. Para não aumentar a probabilidade de haver colisões sucessivas, a cada colisão o tempo de retransmissão poderá ser aumentado de acordo com o algoritmo de backoff exponencial [10]. No caso em que um único nó deseja transmitir, em um dado momento, este nó poderá utilizar toda parte destinada à transmissão de dados. $\mathrm{O}$ agendamento neste esquema é feito pelo nó central, que informará no ACK quantos canais os nós transmissores poderão utilizar. Neste esquema, também, todos os nós sensores deverão estar ativos na parte do sincronismo e na parte de agendamento, mas na parte de transmissão de dados, somente os nós transmissores e receptores ficarão ativos, enquanto que os outros nós ficarão inativos, economizando energia.

\section{ANÁLISE DO CONSUMO DE ENERGIA DO PROTOCOLO PROPOSTO SA-MAC}

Para a análise do protocolo proposto são feitas as seguintes suposições. Na parte de sincronismo do SA-MAC serão reservados 4 bits, e na parte de agendamento serão considerados dois slots de tempo para acesso e dois slots para os ACKs. Para análise do consumo de energia será utilizado um modelo de rádio transmissor TR1000 de 19,2 Kbps, cujo consumo de energia é de $36 \mathrm{~mJ} / \mathrm{s}$ para transmissão, 5,4 a 14,4 mJ/s para recepção e $3 \mu \mathrm{J} / \mathrm{s}$ para o estado de repouso. Serão consideradas duas redes de diferentes tamanhos: 32 e 128 nós sensores. Estes dados são utilizados para o cálculo analítico do consumo de energia do protocolo proposto e que serão comparados com os protocolos S-MAC, T-MAC e FS-MAC apresentados na literatura.

Supõe-se para análise um ciclo de $100 \mathrm{~ms}$ (240 Bytes) para o S-MAC e T-MAC, e um quadro de $100 \mathrm{~ms}$ para os protocolos propostos FS-MAC e SA-MAC. No caso do S-MAC é considerado um período ativo de $20 \mathrm{~ms}$ e um período de repouso de $80 \mathrm{~ms}$. Para o T-MAC o período ativo não é fixo e depende do tráfego, mas para fins de análise será considerado um tempo máximo da parte ativa de $82,5 \mathrm{~ms}$ e um valor de $\mathrm{T}_{\mathrm{A}}$ de $15 \mathrm{~ms}$, 
como usado em [2].

Para o FS-MAC o período ativo depende do número de nós, do número de canais de dados e do tamanho do pacote. Supondo, inicialmente, uma rede de 32 nós sensores, são necessários 5 bits para identificação do nó $\left(2^{5}=32\right.$ nós $)$ e 3 bits para alocação do canal, assim são necessários 8 bits para cada mini-slot, deste modo serão utilizados 256 bits para parte de agendamento do FS-MAC, mais 4 bits destinados a sincronização (totalizando 260 bits ou 32,5 Bytes). Para o cenário descrito, corresponde a $13,55 \mathrm{~ms}$ para a parte de agendamento de acesso do quadro, restando $86,45 \mathrm{~ms}$ para a parte de dados. No caso do protocolo proposto SA-MAC, considerando o mesmo cenário descrito, para um quadro de 100 ms (240 Bytes), 1,67 ms (4 bits de sincronização, 2 Bytes para os slots de acesso, 2 Bytes para os slots de ACK) serão destinados para agendamento de acesso e confirmação do quadro, sendo que o restante, 98,33 ms (236 Bytes) serão destinados a parte de dados.

Para a comparação do consumo de energia dos quatro protocolos, é considerada a transferência de arquivo que contém os seguintes tamanhos: $0,2 \mathrm{~KB}, 0,5 \mathrm{~KB}, 1 \mathrm{~KB}, 5 \mathrm{~KB}$ ou $10 \mathrm{~KB}$. Considerou-se somente o consumo de energia do modo de transmissão do rádio. Neste cenário, para o S-MAC transmitir $0,2 \mathrm{~KB}$ (205 bytes), serão necessários 5 quadros para sinalização (RTS-CTS) e 5 quadros para transmissão de dados. O consumo de transmissão do rádio especificado é de $36 \mathrm{~mJ} / \mathrm{s}$ e o consumo do nó no estado de repouso não será levado em consideração por apresentar um baixo consumo de energia. Considerando o período ativo de $20 \mathrm{~ms}$, consome-se $0,72 \mathrm{~mJ}$ por quadro. Assim, o total do consumo de energia para o S-MAC transmitir um arquivo de $0,2 \mathrm{~KB}$ é 10 quadros $\mathrm{x} 0,72 \mathrm{~mJ}=7,2 \mathrm{~mJ}$.

Para o T-MAC transmitir $0.2 \mathrm{~KB}$ são necessários $2,5 \mathrm{~ms}$ para sinalização (RTS-CTS), 85,31 ms para transmissão dos dados e 15 ms para $\mathrm{T}_{\mathrm{A}}$. O consumo total do T-MAC para transmitir 0,2 $\mathrm{KB}$ é de 4,33 mJ. Para o cenário de uma rede de 32 nós sensores, o FS-MAC na parte de agendamento de acesso utiliza 32,5 bytes, correspondente a 13,55 ms para transmissão, com a capacidade do rádio especificada. Assim, considerando um quadro de $100 \mathrm{~ms}$, restam $86,45 \mathrm{~ms}(100 \mathrm{~ms}-13,55 \mathrm{~ms}=$ $86,45 \mathrm{~ms}$ ) para serem utilizados na transmissão de dados. Desta forma, o consumo total para transmitir $0,2 \mathrm{~KB}$ utilizando o FSMAC é $(13,55 \mathrm{~ms}+85,41 \mathrm{~ms})$ x $0,036 \mathrm{~mJ}=3,56 \mathrm{~mJ}$. Os 85,41 ms é o tempo necessário para transmitir os $0,2 \mathrm{~KB}$ (205 Bytes) de dados.

No caso do protocolo proposto SA-MAC, no cenário de uma rede de 32 nós, são necessários 5 bits para identificação do nó e mais 2 bits para alocação de canais, totalizando 7 bits para slot de tempo de acesso e também para cada slot de tempo de ACK. Como serão utilizados dois slots para agendamento de acesso e dois para confirmação, serão necessários 28 bits, além dos quatro bits de sincronização (totalizando 32 bits, correspondente a 4 bytes). Os 4 bytes correspondem a 1,67 ms que são destinados ao agendamento de acesso, confirmação do quadro (ACK) e sincronização, sendo que o restante, 98,33 ms (236 Bytes) serão destinados a parte de dados. Desta forma como 0,2 KB correspondem a 205 bytes, e o tamanho do quadro que restou para a parte de dados é de 235.5 bytes, será necessário apenas 1 quadro para transmissão deste arquivo. Portanto, o consumo total do SA-MAC para transmitir $0,2 \mathrm{~KB}$ é $(1,67 \mathrm{~ms}+$ $85,41 \mathrm{~ms}) \times 0,036 \mathrm{~mJ}=3,13 \mathrm{~mJ}$. Para os demais tamanhos de arquivos, os consumos de energia podem ser calculados de modo similar, sendo que quanto maior o arquivo, maior o número de quadros necessários para efetuar a transmissão. A Tabela I mostra o consumo de energia para o S-MAC, T-MAC, FS-MAC e do protocolo proposto SA-MAC considerando os diferentes tamanhos de arquivos, para uma rede de 32 nós. A Tabela II mostra o consumo de energia para uma rede 128 nós.

TABELA I

CONSUMO DE ENERGIA DOS PROTOCOLOS PARA TRANSMISSÃO DE DIFERENTES TAMANHOS DE ARQUIVOS - PARA UMA REDE DE 32 NÓS SENSORES.

\begin{tabular}{|l|l|l|l|l|l|}
\hline $\begin{array}{l}\text { Protocolo/ } \\
\text { Tam. Arq. }\end{array}$ & $\mathbf{0 , 2} \mathbf{K B}$ & $\mathbf{0 , 5} \mathbf{~ K B}$ & $\mathbf{1} \mathbf{~ K B}$ & $\mathbf{5} \mathbf{~ K B}$ & $\mathbf{1 0} \mathbf{~ K B}$ \\
\hline S-MAC & $7,20 \mathrm{~mJ}$ & $15,84 \mathrm{~mJ}$ & $31,68 \mathrm{~mJ}$ & $154,08 \mathrm{~mJ}$ & $308,16 \mathrm{~mJ}$ \\
\hline T-MAC & $4,33 \mathrm{~mJ}$ & $9,56 \mathrm{~mJ}$ & $19,14 \mathrm{~mJ}$ & $93,18 \mathrm{~mJ}$ & $186,36 \mathrm{~mJ}$ \\
\hline FS-MAC & $3,56 \mathrm{~mJ}$ & $9,14 \mathrm{~mJ}$ & $17,79 \mathrm{~mJ}$ & $89,00 \mathrm{~mJ}$ & $178,00 \mathrm{~mJ}$ \\
\hline SA-MAC & $3,13 \mathrm{~mJ}$ & $7,86 \mathrm{~mJ}$ & $15,66 \mathrm{~mJ}$ & $78,12 \mathrm{~mJ}$ & $156,24 \mathrm{~mJ}$ \\
\hline
\end{tabular}

TABELA II

CONSUMO DE ENERGIA DOS PROTOCOLOS PARA TRANSMISSÃO DE DIFERENTES TAMANHOS DE ARQUIVOS - PARA UMA REDE DE 128 NÓS SENSORES

\begin{tabular}{|l|l|l|l|l|l|}
\hline $\begin{array}{l}\text { Protocolo / } \\
\text { Tam. Arq. }\end{array}$ & $\mathbf{0 , 2} \mathbf{K B}$ & $\mathbf{0 , 5} \mathbf{K B}$ & $\mathbf{1} \mathbf{~ K B}$ & $\mathbf{5} \mathbf{K B}$ & $\mathbf{1 0 ~ K B}$ \\
\hline S-MAC & $7,2 \mathrm{~mJ}$ & $15,84 \mathrm{~mJ}$ & $31,68 \mathrm{~mJ}$ & $154,08 \mathrm{~mJ}$ & $308,16 \mathrm{~mJ}$ \\
\hline T-MAC & $4,33 \mathrm{~mJ}$ & $9,56 \mathrm{~mJ}$ & $19,14 \mathrm{~mJ}$ & $93,18 \mathrm{~mJ}$ & $186,36 \mathrm{~mJ}$ \\
\hline FS-MAC & $10,29 \mathrm{~mJ}$ & $24,53 \mathrm{~mJ}$ & $46,65 \mathrm{~mJ}$ & $233,27 \mathrm{~mJ}$ & $464,14 \mathrm{~mJ}$ \\
\hline SA-MAC & $3,15 \mathrm{~mJ}$ & $7,90 \mathrm{~mJ}$ & $15,73 \mathrm{~mJ}$ & $78,44 \mathrm{~mJ}$ & $156,89 \mathrm{~mJ}$ \\
\hline
\end{tabular}

Observa-se que para os protocolos S-MAC e T-MAC, diante do cenário proposto, o número de nós sensores na rede não influencia no consumo de energia. O S-MAC tem um alto consumo de energia por ter períodos fixos de transmissão e repouso. O T-MAC e o FS-MAC alcançam um consumo de energia mais eficiente quando comparado ao S-MAC, onde o FS-MAC é mais econômico que o T-MAC para uma rede pequena (32 nós - Tabela I). Se aumentar o número de nós, como é esperado, o FS-MAC é menos eficiente que o T-MAC (128 nós, Tabela II). O FS-MAC, com grande número de sensores e um tamanho do quadro menor, é bastante ineficiente em comparação aos outros protocolos. Isso se deve ao alto overhead, considerando o fato de que o FS-MAC reserva um slot de agendamento de acesso para cada nó da rede. Se aumentar o tamanho do quadro, o FS-MAC melhora a sua eficiência em termos de consumo de energia. Por exemplo, para um quadro de $600 \mathrm{~ms}$, o overhead é menor, pois restará um tempo maior para parte destinada a dados. O SA-MAC é o protocolo que apresenta o menor consumo de energia independente do tamanho da rede e do quadro, quando comparado aos outros protocolos. Na próxima seção apresentase um modelo de análise do atraso do SA-MAC.

\section{ANÁlISE DO ATRASO DOS PACOTES NO SA-MAC}

No protocolo proposto SA-MAC, os pacotes que chegam aos nós sensores são submetidos a dois tipos de atraso. $\mathrm{O}$ primeiro tipo de atraso é devido à solicitação de agendamento para posteriormente transmitir nos slots de dados. Neste caso há uma disputa no canal de acesso com outros nós sensores e pode haver 
retransmissões de solicitação. O segundo tipo de atraso é devido ao esquema de escalonamento no nó sensor central que organiza a seqüência com que os pacotes devem ser transmitidos. A análise desses dois tipos de atraso pode ser feita separadamente. Para a análise serão feitas as seguintes suposições. Os pacotes que são gerados nos nós sensores são esporádicos, diferentes do tráfego de Internet, e serão modelados como chegadas poissonianas de taxa média $\mathrm{G}$ (pacotes/quadro). Para a análise do atraso devido à solicitação de agendamento, será considerado que depois de $r$ quadros o nó sensor saberá se houve sucesso ou falha na transmissão. Para o cálculo de espera devido à retransmissão será levado em conta os parâmetros da Fig. 3.

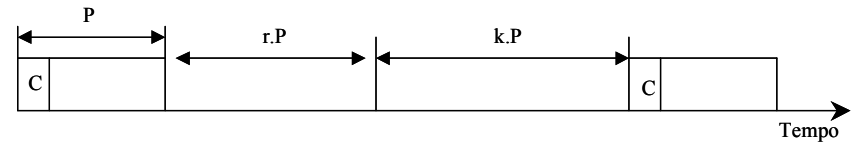

Fig. 3. Diagrama de tempo para análise do tempo de retransmissão.

Na Fig.3, o C representa o tempo de um dos canais de acesso utilizado para transmitir as informações de agendamento em cada quadro de comprimento $\mathrm{P}$, e embora este tempo de transmissão seja pequeno em relação ao quadro, será considerado que o total de transmissão seja $\mathrm{P}$, o tamanho total do quadro, que leva em conta o tempo de processamento e a sua resposta que só ocorre no quadro seguinte. A parte r.P corresponde ao tempo que um nó sensor deve esperar para saber se houve sucesso ou falha na tentativa de acesso e a parte $k . P$ corresponde ao tempo devido à retransmissão (backoff time).

$$
\begin{aligned}
& q_{n}=\left[e^{-G / K}+\frac{G}{K} e^{-G}\right]^{K} e^{-S} \\
& q_{t}=\left[\frac{e^{-G / K}-e^{-G}}{1-e^{-G}}\right]\left[e^{-G / K}+\frac{G}{K} e^{-G}\right]^{K-1} e^{-S}
\end{aligned}
$$

Substituindo as Eqs. 2, 3, 4 e 5 na Eq. 1, obtém-se:

$$
E\{R\}=\left(\frac{1-q_{n}}{q_{t}}\right)\left[r P+\left(\frac{K+1}{2}\right) P\right]
$$

Para slotted Aloha, a vazão S é dada por [11]:

$$
S=G\left[\frac{q_{t}}{1+q_{t}-q_{n}}\right]
$$

Utilizando as expressões acima, o valor $E\{R\}$ pode ser calculado numericamente. $\mathrm{O}$ tempo médio de transferência $\mathrm{T}$, devido somente ao acesso de agendamento, tem quatro componentes: a) tempo de transmissão do pacote de informação, $P ;$ b) o tempo de espera após a chegada do pacote até o início do próximo quadro, $P / 2 ; \mathrm{c}$ ) o atraso devido a retransmissão, $E\{R\} \mathrm{e}$ o atraso de propagação, $\tau$. Assim, $\mathrm{T}$ é dado por:

$$
T=P+P / 2+E\{R\}+\tau
$$

A Tabela III mostra o desempenho do SA-MAC em relação ao atraso de transferência para diferentes valores de taxa de chegada de pacotes em cada nó sensor ( $\lambda$ pac./seg.) e de número de nós sensores (n). $O$ tamanho do quadro $P$ utilizado é $100 \mathrm{~ms}, \mathrm{r}=1$ (significando que o ACK chega no quadro seguinte) e o valor de $\mathrm{K}$ é igual a 5 .

TABELA III

VARIAÇÃO DO TEMPO DE ATRASO DO ESQUEMA DE AGENDAMENTO DE ACESSO DO SA-MAC - G (PAC./P);S (\%) E T (SEG.)

\begin{tabular}{|l|l|l|l|l|l|l|l|l|l|l|l|}
\hline \multicolumn{4}{|c|}{ Taxa Chegada $\boldsymbol{\lambda}=\mathbf{0 , 0 5}$ pac./s } & \multicolumn{3}{|c|}{ Taxa Chegada $\boldsymbol{\lambda}=\mathbf{0 , 1}$ pac/s } & \multicolumn{3}{|c|}{ Taxa Chegada $\boldsymbol{\lambda}=\mathbf{0 , 2}$ pac/s } \\
\hline Nós & $\mathrm{G}$ & $\mathrm{S}$ & $\mathrm{T}$ & Nós & $\mathrm{G})$ & $\mathrm{S}$ & $\mathrm{T}$ & Nós & $\mathrm{G}$ & $\mathrm{S}$ & $\mathrm{T}$ \\
\hline 10 & 0,05 & 0,047 & 0,175 & 10 & 0,1 & 0,088 & 0,201 & 10 & 0,2 & 0,158 & 0,256 \\
\hline 20 & 0,1 & 0,088 & 0,201 & 20 & 0,2 & 0,158 & 0,256 & 20 & 0,4 & 0,254 & 0,379 \\
\hline 30 & 0,15 & 0,125 & 0,228 & 30 & 0,3 & 0,212 & 0,315 & 30 & 0,6 & 0,31 & 0,524 \\
\hline 50 & 0,25 & 0,187 & 0,285 & 50 & 0,5 & 0,286 & 0,448 & 50 & 1,0 & 0,347 & 0,901 \\
\hline 80 & 0,4 & 0,254 & 0,379 & 80 & 0,8 & 0,338 & 0,696 & 80 & 1,6 & 0,312 & 0,1797 \\
\hline 100 & 0,5 & 0,286 & 0,448 & 100 & 1,0 & 0,347 & 0,901 & 100 & 2,0 & 0,267 & 2,747 \\
\hline 200 & 1,0 & 0,347 & 0,901 & 200 & 2,0 & 0,267 & 2,747 & 200 & 4,0 & 0,075 & 20,925 \\
\hline
\end{tabular}

k é uma variável aleatória (inteira) que representa o número de quadros que um nó deve esperar até que possa novamente transmitir no canal de acesso e é dependente da estratégia de backoff. O valor máximo de k é K-1. O atraso de retransmissão pode ser escrito como:

$$
E\{R\}=E\{H\} .(1+r+E\{k\}) \cdot P
$$

onde $E\{H\}$ é o número médio de tentativas de retransmissão. Para uma estratégia de backoff exponencial $E\{k\}$ e $E\{H\}$ são dados por [11]:

$$
\begin{aligned}
& E\{k\}=\frac{K-1}{2} \\
& E\{H\}=\frac{1-q_{n}}{q_{t}}
\end{aligned}
$$

Onde, $q_{n}$ é a probabilidade de sucesso de transmissão, dado que a transmissão é um pacote novo, e $q_{t}$ é a probabilidade de sucesso de transmissão, dado que a transmissão é um pacote de retransmissão. As expressões de $q_{n}$ e $q_{t}$ podem ser calculadas em função de $\mathrm{S}$ (vazão), G e K, e são dadas por [11]:
$\mathrm{O}$ atraso de propagação $\tau$ será desprezado. Para os três diferentes valores da taxa de chegada $\lambda$, apresentados na Tabela III, observa-se que a vazão máxima é alcançada quando os valores de $\mathrm{G}=1$ e $S=0,347$, obtendo-se assim, o número máximo de nós correspondentes na rede que são 200, 100 e 50 . Entretanto, esses valores já estão em pontos de instabilidade. Para operar em regiões estáveis pode-se adotar o seguinte critério de projeto. Como a rede de sensores em estudo necessita um atraso controlado, pode-se limitar o atraso máximo em, por exemplo, 0,5 seg., obtendo-se, neste caso, 100, 50 e 25 nós para as taxas de chegada $0,05,0,1$ e $0,2 \mathrm{pac} / \mathrm{seg}$, respectivamente. Observe-se que neste modelo de análise foi utilizado somente um mini-slot para o acesso de agendamento. Como no esquema do protocolo SA-MAC pode-se utilizar mais de um mini-slot para acesso de agendamento, o número de nós pode ser dobrado para cada aumento de mini-slot. Assim, para taxa de chegada $\lambda=0,1$ e $n=10$, o valor de $G=0,1$ para um minislot, considerando dois mini-slots, $G$ será igual a 0,2 , correspondente a 20 nós. 
Para a análise do segundo tipo de atraso devido ao escalonamento dos pacotes no nó sensor central, pode-se considerar que os buffers de armazenamento dos pacotes estão distribuídos em cada um dos nós sensores e que são atendidos através de $n$ slots de dados de comprimento fixo em cada quadro. Desta forma, como até $n$ nós sensores podem ser atendidos a cada quadro, o modelo de fila $\mathrm{M} / \mathrm{D} / \mathrm{n}$ pode ser utilizado para a estimativa de atraso devido ao escalonamento, considerando um esquema de atendimento FIFO. A expressão exata do tempo de espera para a fila $\mathrm{M} / \mathrm{D} / \mathrm{n}$ é difícil de ser obtida, mas existe uma aproximação dada por Molina, citada em [12] que será adotada:

$$
w \approx \frac{n}{n+1} \cdot E_{2, n}(A) \cdot \frac{h}{n-A} \cdot \frac{1-\left(\frac{A}{n}\right)^{n+1}}{1-\left(\frac{A}{n}\right)^{n}}
$$

onde, $A$ é a intensidade de tráfego total dada em erlangs, $h$ o tempo de serviço e $E_{2, n}(A)$ é a fórmula de Erlang de $2^{a}$ espécie.

Para estimar o atraso de escalonamento foram adotados os seguintes valores: taxa de chegada $\lambda$ de $0,05,0,1,0,2$ e 0,5 pac./seg., um tempo de serviço dado pela diferença de tamanho de quadro $P=0.1 \mathrm{seg}$. e o tempo referente ao acesso de agendamento e de confirmação de 0,00167 (sendo que este tempo varia de acordo com o número de nós), dividido por número de slots de dados ou servidores que foi estipulado em $n$ $=3$. O número de nós varia entre 10 e 150 nós. A Tabela IV apresenta os resultados dos atrasos devido ao escalonamento. Pode-se observar que com os parâmetros dados, os atrasos permanecem pequenos. Entretanto, os atrasos podem-se tornar significativos se houver o aumento de número de nós. Por exemplo, para uma taxa de chegada $\lambda$ de $0,5 \mathrm{pac} / \mathrm{seg}$. e com uma rede de 180 sensores, o atraso pode chegar a $0,5 \mathrm{seg}$. Portanto, este atraso deve ser controlado de acordo com a aplicação específica. $\mathrm{O}$ atraso de escalonamento deve ser somado ao atraso de agendamento para obter o atraso total de um pacote desde a sua chegada até à sua transferência ao destino.

TABELA IV

ATRASO DO ESCALONAMENTO DE TRANSMISSÃO DO SA-MAC

\begin{tabular}{|l|c|c|c|c|}
\cline { 2 - 5 } \multicolumn{1}{c|}{} & $\boldsymbol{\lambda}=\mathbf{0 , 0 5}$ & $\boldsymbol{\lambda}=\mathbf{0 , 1}$ & $\boldsymbol{\lambda = \mathbf { 0 , 2 }}$ & $\boldsymbol{\lambda}=\mathbf{0 , 5}$ \\
\hline Nós & $\mathbf{w}$ & $\mathbf{w}$ & $\mathbf{W}$ & $\mathbf{w}$ \\
\hline 10 & $6,0 \mathrm{E}-09$ & $4,7 \mathrm{E}-08$ & 3,7 E-07 & 5,7 E-06 \\
\hline 20 & 4,7 E-08 & 3,7 E-07 & 2,9 E-06 & 4,3 E-05 \\
\hline 30 & 1,5 E-07 & 1,2 E-06 & 9,7 E-06 & 1,4 E-04 \\
\hline 50 & 7,2 E-07 & 5,6 E-06 & 4,3 E-05 & 6,2 E-04 \\
\hline 80 & 2,9 E-06 & 2,2 E -05 & 1,6 E-04 & 2,6 E-03 \\
\hline 100 & 5,6 E-06 & 4,3 E-05 & 3,2 E-04 & 5,5 E-03 \\
\hline 150 & 1,8 E-05 & 1,3 E-04 & 1,0 E-03 & 3,6 E-02 \\
\hline
\end{tabular}

\section{CONCLUSÕES}

Neste artigo foi proposto um novo protocolo MAC para redes de sensores baseados em TDMA, denominado SA-MAC. É um protocolo voltado para uma aplicação específica, como sistemas médicos, onde os sensores estão altamente concentrados em uma área restrita, apresentando um baixo tráfego e atraso reduzido.
A principal característica do protocolo proposto é o esquema de agendamento de transmissão simplificado, resultando em um baixo consumo de energia e overhead reduzido. O consumo de energia do protocolo SA-MAC comparado ao S-MAC, T-MAC e FS-MAC, mostrou-se mais eficiente, pelo fato de ser mais flexível na forma de se fazer o acesso de agendamento, independente de número de nós da rede.

Quanto ao atraso do protocolo SA-MAC, o principal atraso foi devido acesso de agendamento. Como este atraso é devido à disputa de canal de acesso e a conseqüente retransmissão de pacotes, pode-se definir um limiar máximo de atraso médio e estimar o número de nós para satisfazer um determinado atraso necessário pela aplicação. $\mathrm{O}$ outro atraso devido ao escalonamento no nó central pode ser mantido com valores significativamente baixos controlando o número de nós ou aumentando o número de slots de dados.

\section{REFERÊNCIAS}

[1] W. Ye, J. Heidemann and D. Estrin, "An energy-efficient MAC protocol for wireless sensor networks", in Proceedings of the IEEE International Conference on Computer Communication, pp.1567-1576, USC/Information Sciences Institute, New York, USA, 2002.

[2] T. Van Dam and K. Langendoen, "An adaptive energy-efficient MAC protocol for wireless sensor networks", in Proceedings of the first international conference on Embedded networked sensor systems, pp. 171-180, ACM Press, 2003.

[3] J. Polastre, J. Hill and D. Culler, "Versatile low power media accesss for wireless sensor networks", in Proceedings of the 2 nd international conference on embedded networked sensor systems, pp. 95-107, ACM Press, 2004

[4] R. Kalidindi, L. Ray, R. Kannan and S. Iyengar, "Distributed Energy Aware MAC Layer for wireless sensor networks", in International conference on wireless networks, Las Vegas, Nevada, 2003.

[5] V. Ranjendran, K. Obraczka and J.J.Garcia-Luna-Aceves, "Energyefficient collision-free medium access control for wireless sensor networks", in Proceedings of the first international conference on embedded networked sensor systems, pp.181-192, ACM Press, 2003.

[6] S.S. Oliveira, and S. Motoyama, "Proposal of an MAC protocol for wireless sensor network with fixed scheduling of channels", in Proceedings of the international workshop on telecommunications IWT/07, pp. 178-182, Minas Gerais, Brasil, INATEL, 2007.

[7] W. Ye, J. Heidemann and D. Estrin, "Medium Access Control with Coordinated Adaptive Sleeping for Wireless Sensor Networks", IEEE/ACM Trans. Net., vol. 12, nº. 3, pp. 493-506, June 2004.

[8] S. Kim, D.-S. Huang, X.-P. Zhang and G.-B Huang, "An adaptive energy-efficient and low-delay MAC protocol for wireless sensor networks", ICIC 2005, Part II, LNCS Vol.3645, pp. 598-606, SpringerVerlag Berlin/Heidelberg, 2005.

[9] I. Demirkol, C. Ersoy and F. Alagöz, "MAC Protocols for Wireless Sensor Networks: A Survey", IEEE Communications Magazine, vol. 44, pp. 115-121, April 2006.

[10] D. G. Jeong and W. S. Jeon, "Performance of an exponential backoff scheme for slotted-aloha protocol in local wireless environment," IEEE Transactions on Vehicular Technology, vol. 44, no. 3, August 1995.

[11] J. L Hammond and J.P. O'Reilly. "Performance analysis of local computer networks". Addison-Wesley Publishing Company, pp. 291 299. Inc. 1986.

[12] ITU-D.Handbook - Teletraffic engineering. Study Group 2, question 16/2, pp. 248. Geneva, 2003.

[13] C. Zhihui, A. Khokhar, "Self Organization and Energy Efficient TDMA Mac Protocol by Wake Up for Wireless Sensor Networks", First Annual IEEE Communications Society Conference on Sensor and Ad Hoc Communications and Networks, 2004, pp:335 - 341.

[14] W.L. Lee, A. Datta, R. Cardell-Oliver, "FlexiMAC: A flexible TDMAbased MAC protocol for fault-tolerant and energy-efficient wireless sensor networks", 14th IEEE International Conference on Networks, Sept. 2006, Vol. 2, pp:1-6 